\title{
COMPARATIVE STUDY OF CENTRALIZED AND DECENTRALIZED LOCALIZATION METHODS
}

\author{
Frédéric BALDIT and Jean-Pierre LE CADRE \\ IRISA/CNRS, Campus de Beaulieu, 35042 Rennes Cedex, France.
}

\begin{abstract}
Decentralized Detection (D.D.) is an interesting alternative to classical Centralized Detection (C.D.) when communicationnal/computationnal constraints play an important role in the design of the decision system. In order to promote this new technique, practical demonstration of its feasibility and performance, for realistic situations, has to be done. We present such an application of D.D., in the context of source localization and with Neyman-Pearson (N.P.) criterion, where relatively good performances of D.D. are shown. Of special interest seems to be the inherent robustness of D.D. architectures.
\end{abstract}

\section{INTRODUCTION}

Though it has aroused a considerable literature since the initiatory paper of Tenney and Sandell, [3], D.D. theory yet cannot be considered as an established technique for operationnal distributed decision systems. Among others, usual arguments favouring the use of D.D. systems are the reduction in communicationnal and (on line) computationnal requirements of large systems, especially those with a large number of geographically distributed sensors, but others interesting properties, as fault tolerance and robustness, are also potential abilities of decentralized architectures.

The aim of this paper is, essentially, to investigate the feasability of decentralized techniques for realistic situations, and their relative performance with C.D., when N.-P. criterion is chosen. The paper is organized as follows. After a (qualitative) description of a list of available results from general D.D. theory, we present a first localization model, which comes from the array processing localization field. This model has been extended to its D.D. version. Simulation results are then presented. They confirm that D.D. performances are rather interesting, when compared to the C.D. alternative, in term of some communicationnal cost/detection performance compromise. Other interesting anticipated aspects of D.D. for array processing are its capacity for rejecting local degradations/perturbations of the detection environment. Those have been modeled by a second perturbing source, with a distance-decreasing influence over each array. A second lot of simulations is presented, which confirms that D.D. is a challenging alternative to C.D. for this kind of situation. We finally give concluding remarks and propose future lines of investigation.

This work was supported by our friends of DCAN Toulon

\section{THE GENERAL DECENTRALIZED DETECTION PROBLEM}

Our statistical model is built upon the classical binary hypotheses testing problem. We use the following notations. $E=E_{1} \times E_{2}$ is a measurable space ${ }^{1}, Y=\left(Y_{1}, Y_{2}\right)$ is a random variable (r.v.), where $Y_{i}, i=1,2$ is taking values in $E_{i}, i=1,2$. Probability measures, $P_{0}$ and $P_{1}{ }^{2}$, are respectively associated with two hypotheses, $H_{0}$ and $H_{1}$, such that, under $H_{i}, Y \sim P_{i}, i=0,1$. Observation of $y=Y(\omega)$ is the information gathering process by means of which some decision $U \in\{0,1\}$ has to be made by the statistician concerning the true hypothesis. We assume (for reasons that will be invoked later on) that $P_{i}$ separates, i.e. that $Y_{1}$ and $Y_{2}$ are conditionally independent given any hypothesis. A first caracterization of D.D. is depicted on figure (1). Whereas C.D. elaborates its decision $U=\gamma(Y)$ under observation of the whole measurement $Y$, D.D. imposes each observation $Y_{i}$ to be locally processed and summarized (quantized), $U_{i}=\gamma_{i}\left(Y_{i}\right) \in\{0,1\}$, before being sent to a central data fusion processor, whose output $U_{0}=\gamma_{0}\left(U_{1}, U_{2}\right) \in\{0,1\}$, is the final decision of the system. Thus D.D. essentially imposes functional constraints on the decision making process. In other words, (silent) cooperation is necessary between two decision makers, $D M_{1}$ and $D M_{2}$, who communicate local decisions to a fusion center, $D M_{0}$. One important aspect of the problem formulation is the choice of some optimality criterion, which we next discuss.

\subsection{NEYMAN-PEARSON CRITERION AND RANDOMIZATION ISSUES}

As is usual for many radar/sonar problems, we have supposed that no a priori probabilistic knowledge was available concerning the occurence of the true hypothesis. Thus, choosing N.P. criterion, our optimization problem for the decentralized (respectively centralized) detection problem reduces to finding some decision strategy, $\gamma=\left(\gamma_{0}, \gamma_{1}, \gamma_{2}\right)$, $\gamma_{i}: E_{i} \rightarrow\{0,1\}, i=1,2$ and $\gamma_{0}:\{0,1\}^{2} \rightarrow\{0,1\}$ (respectively some decision rule $\gamma: E \rightarrow\{0,1\})$ maximizing the probability of detection $P_{d}=P\left(\left[U_{0}=1\right] \mid H_{1}\right)$ when the probability of false alarm (f.a.), $P_{f}=P\left(\left[U_{0}=1\right] \mid H_{0}\right)$, is constrained to be no larger than some prefixed rate $\alpha \in$ $(0,1)$. As recalled by N.P. fundamental lemma, [2], solving

\footnotetext{
${ }^{1}$ We assume, whenever not clearly made explicit, that usual measure-theoretic aspects of our definitions are understood.

2 with probability density functions (p.d.f.) $p_{0}$ and $p_{1}$ w.r.t. some dominating $\sigma$-finite measure.
} 
this problem should be done keeping in mind two characteristic properties which are held by optimal (centralized) N.P. tests:

- optimal rules are randomized tests, i.e. are defined by the conditional probability $\phi$ of deciding $H_{1}$ when $y$ is realised: $\phi(y)=P([U=1] \mid Y=y)$,

- any $P_{d}$-maximizing test with size $P_{f}$ is reached among the family of likelihood-ratio tests, i.e. such that

$$
\phi(Y) \stackrel{\text { a.s. }}{=} \begin{cases}1 & \text { if } p_{1}(Y)>t . p_{0}(Y) \\ \delta & \text { if } p_{1}(Y)=t . p_{0}(Y) \\ 0 & \text { if } p_{1}(Y)<t . p_{0}(Y)\end{cases}
$$

where $t \in(0, \infty)$ and $\delta \in(0,1)$ are adjusted in order to reach the size $\alpha$.

For decentralized testing, closed form solutions analog to the C.D. test are generally unavailable ${ }^{3}$. Of particular importance is the above-mentioned conditional independence assumption, without which D.D. leads to hard combinatorial problems, namely NP-complete problems, [4]. Still, making this assumption, person-by-person necessary conditions of optimality ${ }^{4}$ give structural caracterization of the set of optimal decision rules. In this context, particular attention should be paid to the problem of "team-randomization", that is extension of the classical randomization mechanism to many decision makers. More precisely, one can show that, under certain configurations, it may be necessary for the different $D M$ is to coordinate their hesitation in the decision making process. In order to present some useful results, some definitions are needed. We first define deterministic strategies, which are those never using randomized decision rules, that is those for which our former notation $\gamma=\left(\gamma_{0}, \gamma_{1}, \gamma_{2}\right)$ is correct $^{5}$. Second, we define the convex combination $(p, q)$ of the deterministic strategies $\gamma^{(1)}$ and $\gamma^{(2)}$ as the randomized strategy that consists in using $\gamma^{(1)}$ and $\gamma^{(2)}$ with respective probability $p$ and $q$, where $p, q \geq 0, p+q=1$. Then the following two results are available:

1. When N.P. optimization problem is defined over the set of deterministic strategies, and when distribution of $Y_{i}, i=1,2$ contains no point masses, one can restrict attention to (non randomized) likelihood-ratio strategies for $D M_{1}, D M_{2}$,

2. every N.P. optimal strategy (when defined over the most general set of randomized strategies), can be defined (reached) as a convex combination of two deterministic strategies.

Though they may seem useless for some practical design of optimal strategies, the preceding two results can be combined in order to construct decentralized ROC curves, as is shown in next section.

\footnotetext{
${ }^{3}$ We refer the reader to $[5],[1]$ for a more comprehensive formal treatment of D.D. theory.

${ }^{4}$ That is optimization of one decision maker when the rest of the team is fixed.

${ }^{5}$ Note that for such a strategy a boolean fusion rule as to be chosen. We refer to [1] for the choice of this rule, which can be restricted to monotone rules
}

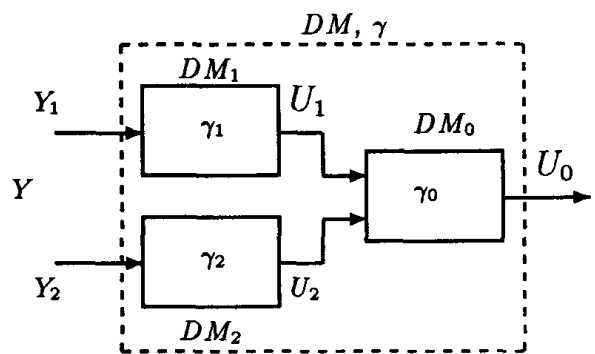

Figure 1: D.D. and C.D. (dashed) architectures.

\subsection{PRACTICAL SEARCH OF N.P.-OPTIMAL STRATEGIES}

Before using the above results, we need to show some tight connexion between N.P. and Bayes criteria. Using Bayes approach with the error probability criterion ${ }^{6}$, and noting that N.P. constrained maximization problem can be reformulated $^{7}$ as the minimization (w.r.t. to some randomized strategy $\gamma$ ) of $\lambda . P_{f}-P_{d}$ for some Lagrange multiplier $\lambda \geq 0$, one easily sees that any Bayes-optimal decentralized (deterministic) strategy with priors $\pi_{i}=P\left(\left[H=H_{i}\right]\right), i=$ 0,1 such that $\lambda=\pi_{0} / \pi_{1}$ is N.P.-optimal. For this reason, whenever needed, one disposes of a simple way for ploting deterministic decentralized ROC curves. Namely, that consisting in varying parameter $\lambda$ in $[0, \infty]$ and solving all the associated Bayesian problems. For every such problem, knowing that optimal D.D. Bayesian strategies are deterministic (which is another available result of D.D. theory), one is ensured, by point 1) above, that only two thresholds, $t_{1}$ and $t_{2}$, and a fusion rule $\gamma_{0}$ have to be found. We can now fully use the previous points. As all randomized optimal strategies are convex combinations of deterministic strategies, it follows that the optimal decentralized ROC curve is simply the convex hull of the different deterministic ROC curves described in the previous reasonning. Applying this yields the complete N.P. characterization of D.D. performances and, above all, is a method for avoiding the person-by-person principle which is unpractical in the N.P. context. We will discuss in the remaining of the paper the implications of our method for determining whether randomization is necesary. We now present the concrete decentralized localization models.

\section{TWO SIMPLE LOCALIZATION MODELS}

Our first array processing localization model is the following. We assume a far field situation, one point source $S$, with mean power $\sigma(>0)$, and two uniform linear arrays $A_{i}$, composed of $K_{i}, i=1,2$ sensors. $S$ is located at bearing $\theta_{i}$ w.r.t. $A_{i}$. A Gaussian spatially white ambient noise with mean power normalized to one is supposed, independent of $S$. With half-wavelength spatial sampling of the impinging plane waves, raw sensor outputs are transformed to their

\footnotetext{
${ }^{6}$ Between the final decision and the true hypothesis.
}

${ }^{7}$ as the set of randomized strategies is convex. 
equivalent discrete Fourier transform at frequency $f^{8}$, the usual snapshots, $X_{i, n} \in \mathbb{C}^{K_{i}}, n=1, \ldots, N_{i}$ on array $A_{i}$. We assume that the snapshots are i.i.d. $C N\left(0, \Gamma_{i}\right)$, with p.d.f. $p_{i}$ and complex covariance (interspectral) matrix $\Gamma_{i}$, hermitian positive definite, such that ${ }^{9}$

$$
p_{i}(x)=\pi^{-K_{i}} \cdot\left|\Gamma_{i}\right|^{-1} \cdot \exp \left\{-x^{*} \Gamma_{i}^{-1} x\right\}, i=1,2 .
$$

Recalling our previous definitions, we can state the binary hypotheses model as

$$
X_{i, n} \stackrel{H_{j}}{\sim} C N\left(0, \Gamma_{i, j}\right), \quad j=0,1 ; i=1,2 ; n=1, \ldots, N_{i}
$$

with

$$
\left\{\begin{array}{l}
H_{0}: \Gamma_{i, 0}=I_{i} \\
H_{1}: \Gamma_{i, 1}=\Gamma_{i, 0}+\sigma . d_{i} d_{i}^{*}
\end{array}\right.
$$

and

$$
d_{i}^{\prime}=\left[1, e^{-i \pi \cos \theta_{i}}, \ldots, e^{-i \pi \cos (K-1) \theta_{i}}\right] .
$$

As indicated by classical C.D. theory, the locally optimal test on $A_{i}$ is

$$
\left.\Delta_{i}=\frac{1}{N_{i}} \cdot \sum_{n=1}^{N_{i}}\left|X_{i, n}^{*} d_{i}\right|^{2}\right) \underset{U_{i}=0}{\gtrless} t_{i},
$$

where $t_{i}$ is adjusted to satisfy the false alarm constraint. $\Delta_{i}$ 's p.d.f. is obtained through computation of its characteristic function (c.f.), which appears to be the Fourier transform of some $G(a, b)$ (Gamma) probability. More precisely, one can show that, with the i.i.d. assumption,

$$
\Delta_{i} \begin{cases}\stackrel{H_{\mathfrak{O}}}{\sim} & G\left(N_{i}, \alpha_{i}\right) \\ \underset{H_{1}}{\sim} & G\left(N_{i}, \beta_{i}\right)\end{cases}
$$

with

$$
\begin{aligned}
\alpha_{i} & =K_{i} / N_{i} \\
\beta_{i} & =K_{i} \cdot\left(1+K_{i} \cdot \sigma^{2}\right) / N_{i} .
\end{aligned}
$$

Concerning the optimal centralized test, we can easily derive the l.r.t.

$$
\Delta=C_{1} \cdot \Delta_{1}+C_{2} \cdot \Delta_{2} \underset{U=0}{U=1} t,
$$

where coefficients $C_{1}, C_{2}$ are given by

$$
C_{i}=\sigma^{2} \cdot N_{i} /\left(1+K_{i} \cdot \sigma^{2}\right), i=1,2 .
$$

The statistic of $\Delta$, sum of two independant Gamma r.v.s, is approximated by a Gamma distribution. This approximation confirmed to be very good when compared to the real one, which is a linear combination of Gamma r.v.s. Finally, the cumulative distribution function of a Gamma r.v. is needed, in order to compute the various probabilities

\footnotetext{
${ }^{8}$ Omitted in the sequel.

${ }^{9} x^{\prime}, x^{*}$ are respectively the transpose and transpose-complex conjugate of $x$.
}

of false alarm and detection. Those are obtained in closed form as

$$
P_{f}(t)=\int_{t}^{\infty} p_{\Delta}(\delta) d \delta=e^{-t / \alpha} \cdot \sum_{n=0}^{N-1} \frac{1}{n !}\left(\frac{t}{\alpha}\right)^{n}
$$

with an analogous formula holding for $P_{d}(t)$. Note that $\Delta_{i}$ 's p.d.f. is independent of $\theta_{i}$. In fact, our first model could have equivalently be written with a single bearing, which is more consistant with the far field hypothesis. This is due to the a priori knowledge of the bearing of the source, and to the special structure of our physical wave propagation and geometric model.

In a second approach, our first model has been extended to a two-sources version, as is now described. We simply add a second perturbing source, now denoted as $S_{1}$, whose power is modeled as range-decreasing (with transmission loss in $20 . \log (r)$ ), in order to essentially radiate its energy to one of the two arrays. Equations (1),(2) now become

$$
\left\{\begin{array}{l}
H_{1}: \Gamma_{i, 1}=I_{i}+\sigma_{1, i} \cdot d_{1, i} d_{1, i}^{*} \\
H_{2}: \Gamma_{i, 2}=\Gamma_{i, 1}+\sigma_{2} \cdot d_{2} d_{2}^{*}
\end{array}\right.
$$

$\sigma_{1, i}$ and $d_{1, i}$ are respectively the power of $S_{1}$ received at $A_{1}$ and the stearing vector of $S_{1}$ when viewed from $A_{i}, d_{2}$ is the (single) stearing vector associated with $S_{2}$. See [1] for a geometric description of this model. Algebraic computations yield, in a way comparable to the one source case, new expressions for $\Delta_{i}, i=1,2$ and their c.f.s under hypotheses $H_{1}, H_{2}$. Those lead to conclude that the Gamma-character of the p.d.f.s is conserved, but with new parameters, which are now dependent of the bearings with which $S_{1}$ is perceived by $A_{i}$, denoted $\theta_{1, i}$. The local structure of l.r.t. is slithly modified, so that (1) rewrites

$$
\left.\Delta_{i}^{\prime}=\frac{1}{N_{i}} \cdot \sum_{n=1}^{N_{i}}\left|X_{i, n}^{*} d_{2, i}^{\prime}\right|^{2}\right) \underset{U_{i}=0}{\gtrless} t_{i},
$$

with

$$
d_{2, i}^{\prime}=\Gamma_{1, i}^{-1} d_{2, i}
$$

Equations (2)-(8) find corresponding versions for the twosources case, described in ([1]). We now describe some simulation results corresponding to those models.

\section{SIMULATION RESULTS}

As a first result, using the procedure of section (2.2), we have compared R.O.C. curves of C.D. and D.D. for various signal/noise environments. Figure (1) illustrates the behaviour of D.D. with a specific setlement of the various parameters defining our first localization model. It appears that, for almost every configurations, the deterministic R.O.C. curve (defined as the upper envelope of the two candidate "OR" and "AND" fusion rules) is already almost concave (except for very low f.a. rate), so that randomization could be neglected without important loss. If necessary, randomization could be realised, but we remind that this would amount to coordinate different local random mechanisms. In short, randomization could be carried out by letting every $D M_{i}, i=0,1,2 \mathrm{flip}$ a same coin for each

\section{IV-71}




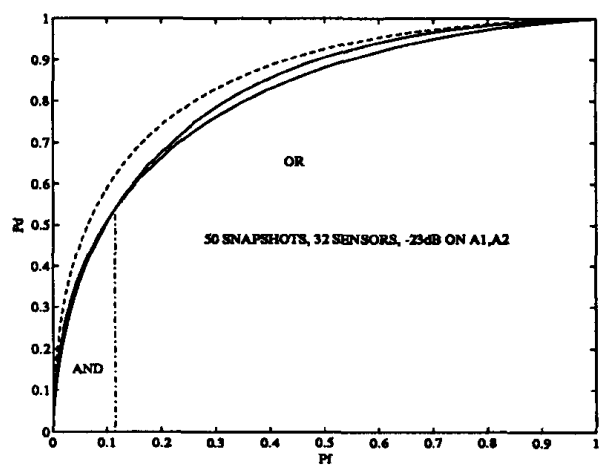

Figure 2: C.D. (dashed) and D.D. with a single source model.

decision, and decide to choose "OR" or "AND" rule ${ }^{10}$. We have observed that D.D. offers R.O.C. that are relatively interesting when compared to the communicationnal reduction. For instance, if the distance between C.D. and D.D. curves is measured in Signal to Noise Ratio (S.N.R.) dBs, it was approximately of $.5 \mathrm{~dB}$ for figure (2). Also interesting is the fact that this difference seems quite insensitive to the S.N.R. itself. Others simulations with three arrays provide the same kind of results.

As a second result, we have also computed the respective performances of D.D. and C.D. when the correct twosources model is taken into account in the detection procedure. Analogous results (i.e. small loss) to the one source model have been observed. It is interesting to note (though not surprising) that both C.D. and D.D. behave relatively well as long as the two interfering sources have sufficiently different bearings, independently from $S_{1}$ power.

As a final result, we have experimented the ability of D.D. to reject local perturbations. Those where modeled by our second localization model, when the decision system is unaware of this model mismatch. Note that this situation can either be interpreted as a bad modelization, or as faulty operation of the system. Figure (3) confirms that D.D. is robust when faced to such problems, as it yields performances that become better than C.D. for small perturbations ${ }^{11}$. In our example, local S.N.R. of $A_{2}$ was lowered by $1 \mathrm{~dB}$ with noisy source $S_{1}$, whereas it was almost unchanged on $A_{1}$. What should be also precised here, is that degrading the situation by model mismatch leads the decision system to work with real f.a. rates that are different from the one expected. It is also anticipated here that D.D. behaves better than C.D. w.r.t. this aspect, that is degrades less the actual $P_{f}$ than does C.D.. This would necessitate further investigation.

\footnotetext{
${ }^{10}$ See [1]. Note that this means, for $D M_{i}, i=1,2$, to choose his threslhold $t_{i}$ between a pair $\left(t_{i}, \mathrm{OR}, t_{i}\right.$ AND $)$ of two thresholds.

${ }^{11}$ Note that "AND" rule becomes better (more robust) than "OR" in this case. In fact this rule could be chosen, as it is comparable to the "OR" rule in the correct model case.
}

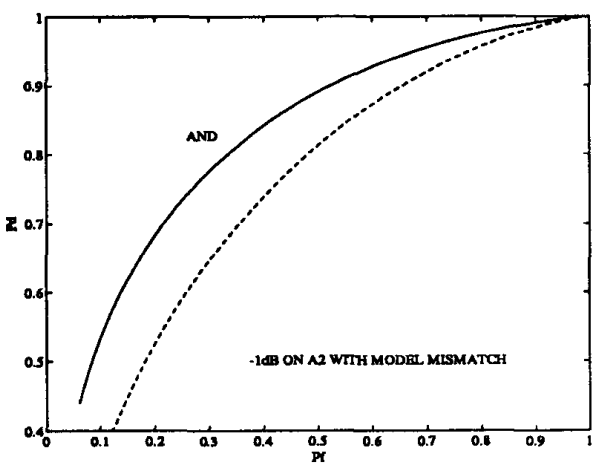

Figure 3: C.D. (dashed) and D.D. with model mismatch by a two sources model.

\section{CONCLUSION AND PROSPECTIVE REMARKS}

Concrete results concerning the use of D.D. for an array processing situation have been obtained. Though certainly specific to the particular problem investigated, simulation results show that

- Some (important) communicationnal complexity reduction is obtained at the cost of some (moderate) performance loss,

- this matter of fact remains apparently true for a large class of (S.N.R.) configurations,

- above all, D.D. seems offering a challenging alternative to C.D. when robustness is an important design criterion.

Further studies would investigate those properties for more than two arrays, and, in general for more complex detection contexts. Special interest to the feasibility of D.D. when a large number of local decision-makers are to cooperate is our present domain of interest.

\section{REFERENCES}

[1] Baldit, F., Le Cadre, J.P., "Decentralized Detection: a survey," Internal Report of the IRISA, 1992, to be published.

[2] LehmanN, E.L., "Testing Statistical Hypotheses," J.Wiley \& Sons eds., 1959.

[3] Tenney, R.R., Sandell, N.R., "Detection with Distributed Sensors," IEEE Trans. on A.E.S., Vol.17, No.4, 1981, pp.641-644.

[4] Tsitsiklis, J.N., Athans, M., "On the Complexity of Decentralized Decision Making and Detection Problems," IEEE Trans. on A.C., Vol.30, No.5, 1985, pp.440446.

[5] Tsitsiklis, J.N., "Decentralized Detection," in Advances in Statistical Signal Processing, Vol.2: Signal Detection, Poor, H.V. and Thomas, J., eds., Greenwich, CT: Jai Press, 1991. 